\title{
A PROBLEMÁTICA AMBIENTAL DA CONTAMINAÇÃO DOS RECURSOS HÍDRICOS POR FÁRMACOS
}

THE ENVIRONMENTAL PROBLEM OF CONTAMINATION OF WATER RESOURCES BY PHARMACEUTICALS

\section{Mayara Andria \\ da Silva Escher (1)}

Especialista em Gerenciamento Ambiental pela Escola Superior de Agricultura Luiz de Queiroz, da Universidade de São Paulo (ESALQ/ USP) - Piracicaba (SP), Brasil.

\section{Juliana Heloisa Pinê}

\section{Américo-Pinheiro (1)}

Professora titular do Programa de Pós-Graduação em Ciências Ambientais da Universidade Brasil (UNIVBRASIL) - São Paulo (SP), Brasil.

\section{Nádia Hortense Torres (1)}

Pós-doutoranda no Laboratório de Tratamento de Resíduos e Efluentes (LTRE) do Instituto de Tecnologia e Pesquisa (ITP) da Universidade Tiradentes (Unit) - Aracaju (SE), Brasil.

\section{Luiz Fernando}

\section{Romanholo Ferreira}

Professor doutor do Programa de Pós-Graduação em Engenharia de Processos no ITP da Unit - Aracaju (SE), Brasil.

\section{Endereço para correspondência:} Mayara Andria da Silva Escher Rua Primavera, 120-CentroCEP 85420-000-Corbélia (PR), BrasilE-mail:mayaraandria@hotmail.com

Recebido em: 03/05/2019

Aceito em: 27/06/2019

\section{RESUMO}

A degradação dos recursos hídricos e a escassez de água potável são assuntos de crescente preocupação mundial, principalmente em razão da presença de contaminantes emergentes (CE) como os fármacos. O presente trabalho teve como objetivo levantar informações por meio de estudos publicados sobre a presença de fármacos em matrizes ambientais e os seus efeitos no meio aquático, assim como para os organismos aquáticos e os seres humanos expostos. Observou-se aumento no número de pesquisas, tanto daquelas voltadas à contaminação dos recursos hídricos por fármacos quanto daquelas voltadas aos efeitos desses nos seres vivos. Verificouse que os fármacos estudados (diclofenaco, ibuprofeno e paracetamol) podem ser encontrados em diversas matrizes ambientais e causar efeitos ecotoxicológicos a diversos organismos não alvos como algas, moluscos, peixes e plantas aquáticas. Para os contaminantes estudados, não existe legislação associada à sua presença em matrizes ambientais, no entanto a União Europeia (UE) inseriu o diclofenaco na lista dos contaminantes que devem ser monitorados. A problemática ambiental da contaminação dos recursos hídricos pelos fármacos envolve grandes desafios, entre eles a busca por medidas que visem à prevenção de possíveis impactos ao meio ambiente e à saúde humana.

Palavras-chave: anti-inflamatórios; diclofenaco; saúde; toxicidade.

\section{ABSTRACT}

Degradation of water resources and the scarcity of drinking water are a matter of growing concern worldwide, mainly due to the presence of emerging contaminants (EC) such as pharmaceuticals. The aim of the present study was to obtain information through published studies on the presence of drugs in environmental matrices and their effects on the aquatic environment, as well as on aquatic organisms and exposed humans. There was an increase in the number of researches, both those focused on the contamination of water resources by pharmaceuticals, and those focused on their effects on living beings. It has been observed that the pharmaceuticals studied (diclofenac, ibuprofen and paracetamol) can be found in several environmental matrices and may cause ecotoxicological effects on several non-target organisms such as algae, molluscs, fish and aquatic plants. For the pollutants studied, there is no legislation associated with their presence in environmental matrices, however the European Union (EU) has already inserted diclofenac in the list of contaminants that should be monitored. The environmental problem of contamination of water resources by pharmaceuticals involves major challenges, among them the search for measures aimed at preventing possible impacts on the environment and human health.

Keywords: anti-inflammatory agents; diclofenac; health; toxicity. 


\section{INTRODUÇÃO}

A qualidade da água é um assunto de crescente preocupação, principalmente por conta da presença de contaminantes emergentes (CE) no ambiente aquático. Após a Segunda Guerra Mundial, o desenvolvimento de novas tecnologias e em consequência a maior quantidade de recursos a serem explorados, alterou a qualidade dos recursos hídricos em razão dos resíduos despejados sem o tratamento necessário, entre eles os fármacos e os produtos de cuidados pessoais (PCPs).

O aumento da expectativa de vida dos seres humanos e a consequente inversão das pirâmides de idade populacional têm aumentado de maneira proporcional o consumo de medicamentos, sendo estes responsáveis diretos pelo incremento na produção e no consumo de fármacos e PCPs (WILKINSON et al., 2015).

Essas substâncias químicas são consumidas e atingem diariamente o meio ambiente. A presença desses compostos em matrizes ambientais como água e solo podem causar efeitos tóxicos nos seres vivos, como genotoxicidade, perturbação endócrina e seleção de bactérias patogênicas resistentes (KÜMMERER, 2010), além da sua persistência no ambiente, potencial eco- toxicológico e prejuízos à saúde dos seres humanos (BISOGNIN; WOLFF; CARISSIMI, 2018). Pesquisas têm detectado compostos farmacológicos, cosméticos e produtos de higiene pessoal em águas superficiais, subterrâneas, água para consumo humano e, até mesmo, em solos sujeitos à aplicação de lodo de esgoto (FENT; WESTON; CAMINADA, 2006).

Existem vários tipos de CE que são preocupantes quando presentes no ambiente, como os fármacos, que são encontrados em ambientes aquáticos. Entre eles, destacam-se os analgésicos e os anti-inflamatórios não esteroides (AINEs) como o diclofenaco (KHETAN; COLLINS, 2007; SANTOS et al., 2010). Esses contaminantes podem trazer prejuízos ao meio ambiente por causa da sua persistência em matrizes ambientais e possíveis alterações no sistema endócrino dos seres vivos, principalmente da fauna aquática (GHISELLI; JARDIM, 2007).

Objetivou-se levantar informações sobre a presença de fármacos em matrizes ambientais, seus efeitos no meio aquático e suas consequências para os organismos aquáticos e para os seres humanos expostos.

\section{CONTAMINAÇÃO DOS RECURSOS HÍDRICOS POR FÁRMACOS E SEUS IMPACTOS NOS SERES VIVOS}

A classe de fármacos mais consumida corresponde a dos analgésicos e anti-inflamatórios (ZHANG; GEISSEN; GAL, 2008; AMÉRICO-PINHEIRO et al., 2017). Isso se deve ao fato de que na maioria dos países os anti-inflamatórios não precisam de prescrições médicas para serem adquiridos, além de serem os mais prescritos por médicos e dentistas (BISOGNIN; WOLFF; CARISSI$\mathrm{MI}, 2018)$. Esses medicamentos sem prescrição podem acarretar diversas consequências, sobretudo quando descartados de maneira inadequada, sendo o destino final o lixo comum.

A presença de fármacos no ambiente foi relatada pela primeira vez por Garrison et al. (1976), que identificaram o ácido clofíbrico em efluente tratado nos Estados Unidos, na faixa de 0,8 a $2,0 \mu \mathrm{g} / \mathrm{L}$. Os estudos sobre a ocorrência desses contaminantes no meio ambiente vêm crescendo e alertam sobre o seu possível risco tóxico, que, apesar de se apresentarem em baixas concentrações, têm recarga contínua. O progressivo aumento da presença de fármacos nos mananciais de abastecimento representa uma das problemáticas mundiais em relação ao comprometimento da qualidade das águas destinadas para o consumo humano e aos prejuízos inerentes aos ambientes aquáticos (AMÉRICO et al., 2012).

Em grandes cidades, as vias de exposição com maior relevância para o ambiente são os compostos farmacológicos de uso humano, enquanto em regiões com atividades agropecuárias e de aquicultura, as principais vias são as substâncias de uso veterinário. Os fármacos não são completamente degradados após sua utilização e atingem o ciclo da água por meio de distintas rotas. As Estações de Tratamento de Esgoto (ETEs) são as principais vias de entrada dos fármacos nos corpos d'água, enquanto a maioria dos resíduos desses compostos de uso veterinário é liberada diretamente no ecossistema pela excreção animal (BILA; DEZOTTI, 2003). 
Estudos revelaram que as substâncias presentes nos fármacos são persistentes no ambiente e não são efetivamente removidas pelos tratamentos convencionais nas ETEs (AMÉRICO et al., 2012). Os principais medicamentos encontrados no ambiente são os analgésicos, anti-inflamatórios, $\beta$-bloqueadores, reguladores lipídicos, antiepiléticos, antidepressivos, hormônios e esteroides, antibióticos e antineoplásicos. Esses dois últimos são uma das classes com maior potencial para causar efeitos negativos no ambiente, pois são citotóxicos, danificando o DNA, inibindo a sua síntese e interrompendo a replicação celular (SANTOS, 2014).

Há preocupação com os possíveis efeitos desses compostos em organismos aquáticos, bem como com os elos da cadeia trófica que podem alimentar-se desses seres vivos contaminados, especialmente porque o ser humano se encontra no topo dessa cadeia (TORRES et al., 2012). Em concentrações na ordem de $n g / L$, os fármacos podem alterar o sistema endócrino dos organismos aquáticos, desencadeando efeitos adversos como a interferência no crescimento, desenvolvimento e/ou reprodução (CUNHA et al., 2017).

A presença de fármacos em águas superficiais, subterrâneas, de consumo humano e em sedimentos foi relatada em vários estudos (STUMPF et al., 1999; AMÉRICO-PINHEIRO et al., 2017). No fim da década de 1990 foram publicados os primeiros artigos sobre a ocorrência, o destino e o efeito dos compostos farmacológicos no ambiente. Essas pesquisas indicam a presença desses contaminantes e seus metabólitos no meio ambiente, principalmente nos recursos hídricos, em concentrações na ordem de ng/L e $\mu \mathrm{g} / \mathrm{L}$ (BILA; DEZOTTI, 2003). Inicialmente, as pesquisas eram voltadas para a detecção desses compostos. Mais tarde, tornou-se intensa a investigação sobre o seu destino e seus efeitos ecotoxicológicos.

Os relatos sobre os impactos ambientais de fármacos são escassos no Brasil. Os estudos realizados se concentram na deteç̧ão, remoção ou nos efeitos tóxicos dessas substâncias no ambiente. O ibuprofeno e o diclofenaco são os anti-inflamatórios mais reportados na literatura (SANTOS et al., 2010; AMÉRICO-PINHEIRO et al., 2017). Por esse motivo, nesse artigo foram discutidos os fármacos classificados como AINEs mais abordados na literatura, como o diclofenaco, o ibupro- feno e o paracetamol, comumente presentes em águas superficiais e ETEs (AMÉRICO et al., 2012; CAMPANHA et al., 2015; KRAMER et al., 2015).

Comparando-se com as pesquisas internacionais, no Brasil existem poucas informações sobre a ocorrência de fármacos no meio ambiente e nas estações de tratamento de água e esgoto. A Tabela 1 apresenta estudos sobre o tema no país, destacando os AINEs presentes em esgoto sanitário, efluentes de ETE e águas superficiais. A presença desses compostos no ambiente é associada às condições sanitárias dos corpos hídricos que recebem efluentes sanitários (STUMPF et al., 1999; ALMEIDA; WEBER, 2006).

Trabalhos sobre a toxidade do diclofenaco mostram que esse composto pode apresentar efeitos mutagênicos sobre microrganismos. Ele está entre os anti-inflamatórios mais tóxicos em estudos de exposição aguda (FENT; WESTON; CAMINADA, 2006). Ghelfi (2014) avaliou os efeitos agudos tóxicos de diclofenaco no ambiente aquático, por meio de bioensaio com o peixe Rhamdia quelen (Jundiá), após 96 horas de exposição nas concentrações de 2 e $20 \mu \mathrm{g} / \mathrm{L}$ observou-se alterações hematológicas e renais nessa espécie.

Hoeger et al. (2005) em um estudo com truta marrom (Salmo trutta $f$. fario), peixe nativo dos rios alemães, exposta a concentrações de diclofenaco semelhantes às registradas em ambientes aquáticos, observaram alterações no fígado e na integridade das brânquias dos animais. Em estudos realizados a fim de avaliar os efeitos de produtos farmacêuticos, entre eles o diclofenaco, em truta arco-íris (Oncorhynchus mykiss) e carpa comum (Cyprinus carpio), observou-se que o diclofenaco causa alterações no fígado, rins e brânquias dessas espécies (TRIEBSKORN et al., 2007), além do aumento da peroxidação lipídica em mexilhões zebra (Dreissena polymorpha) expostos a concentrações de $1 \mu \mathrm{g} / \mathrm{L}$ (QUINN et al., 2011).

O ibuprofeno é um analgésico e anti-inflamatório com maior prescrição no tratamento de dores reumáticas e febres, sendo a terceira droga mais popular no mundo (ALMEIDA; WEBER, 2006). Ensaios laboratoriais com o peixe Oreochromis niloticus (tilápia) exposto a $300 \mathrm{ng} / \mathrm{L}$ de ibuprofeno em ensaios de toxicidade aguda (48 h) e subcrônicos (10 dias) demonstraram o efeito genotóxico desse anti-in- 
flamatório. Observou-se risco ambiental aquático desse contaminante ao se verificar frequências superiores de micronúcleos nos eritrócitos dos peixes submetidos aos testes subcrônicos (RAGUGNETTI et al., 2011). Estudos com o molusco Dreissena polymorpha demonstraram que o ibuprofeno nas concentrações de 0,2-0,8 $\mu \mathrm{g} / \mathrm{L}$ pode causar alterações genéticas em curto período de exposição (PAROLINI; BINELLI; PROVINI, 2011).

O paracetamol é um analgésico e antipirético muito utilizado no Brasil. Autores reportaram a presença do fármaco em águas superficiais (ALMEIDA; WEBER, 2006; CAMPANHA et al., 2015) e em efluente de ETE (AMÉRICO et al., 2012). Esse composto, na con- centração de $100 \mu \mathrm{g} / \mathrm{L}$, causou redução na atividade enzimática da catalase em carpas (Cyprinus carpio) expostas ao fármaco durante 96 horas. Os metabólitos reativos formados durante o metabolismo desse composto causam danos diretos às enzimas, oxidando-as e alterando a sua conformação, fazendo com que percam sua atividade enzimática (NAVA-ÁLVAREZ et al., 2014). Exposições crônicas ao paracetamol (21 dias) podem causar efeitos pro-oxidantes em peixes da espécie Rhamdia quelen nas concentrações de 0,25 e $2,5 \mu \mathrm{g} / \mathrm{L}$. Os peixes expostos apresentam aumento na atividade de superóxido dismutase, uma resposta relacionada ao estresse oxidativo (GUILOSKI et al., 2017).

\section{Tabela 1 - Ocorrência e concentração de fármacos anti-inflamatórios não esteroides (AINEs) em matrizes ambientais em diferentes estados brasileiros.}

\begin{tabular}{|c|c|c|c|}
\hline Referência & Fármaco & Matriz/estado & Concentração (ng/L) \\
\hline \multirow{2}{*}{ Stumpf et al. (1999) } & \multirow{7}{*}{ Diclofenaco } & Água Superficial/RJ & $20-40$ \\
\hline & & Efluente de ETE/RJ & $100-1.000$ \\
\hline Almeida; Weber (2006) & & Água Superficial/SP & $8,1-394,5$ \\
\hline Américo et al. (2012) & & Esgoto Bruto/ETE/MS & 2.471 .000 \\
\hline Kramer et al. (2015) & & Água superficial/PR & 285 \\
\hline Stelato et al. (2016) & & Água Superficial/SP & $2.800-10.900$ \\
\hline Américo-Pinheiro et al. (2017) & & Água Superficial/MS & $120-8.250$ \\
\hline Stumpf et al. (1999) & \multirow{5}{*}{ Ibuprofeno } & Água Superficial/RJ & $<10$ \\
\hline Almeida; Weber (2006) & & Água Superficial/SP & $10,0-78,2$ \\
\hline Américo et al. (2012) & & Esgoto Bruto/ETE/MS & 2.325 .000 \\
\hline Kramer et al. (2015) & & Água superficial/PR & 370 \\
\hline Stelato et al. (2016) & & Água Superficial/SP & $1,4-4,2$ \\
\hline Almeida; Weber (2006) & \multirow{5}{*}{ Paracetamol } & Água Superficial/SP & $0,3-10,3$ \\
\hline Américo et al. (2012) & & Esgoto Bruto/ETE/MS & 130.000 \\
\hline Campanha et al. (2015) & & Água Superficial/SP & $104,7-13496$ \\
\hline Kramer et al. (2015) & & Água superficial/PR & 261 \\
\hline Stelato et al. (2016) & & Água Superficial/SP & $228.000-590.000$ \\
\hline
\end{tabular}

ETE: estação de tratamento de esgoto. 


\section{ASPECTOS LEGAIS}

No Brasil, não há registros de programas oficiais voltados para a problemática dos fármacos como contaminantes. Pesquisas têm contribuído fornecendo subsídios para tomadas de decisões e despertando o interesse de empresas de saneamento, órgãos governamentais e a população em geral. O mais preocupante é que não há uma legislação que determina limites de lançamento de fármacos no meio ambiente.

Há setores e legislações brasileiras sobre a qualidade da água, porém a Resolução do Conselho Nacional de Meio Ambiente (CONAMA) no 357/2005 (padrões de classificação de corpos de água), a Resolução no 396/2008 (enquadramento das águas subterrâneas) e a Resolução CONAMA no 430/2011 (lançamento de efluente) não dispõem de valores limites para os compostos farmacológicos (BRASIL, 2005; 2008; 2011).

A Agência de Proteção Ambiental dos Estados Unidos (USEPA) publica diretrizes e leis sobre matrizes ambientais, entretanto, a legislação americana não estabelece os limites de concentração para fármacos em corpos de água. A USEPA apresentou quatro listas de contaminantes emergentes candidatos à futura regulamentação, na quarta atualização da lista estão incluídos 97 produtos químicos e 12 contaminantes biológicos (USEPA, 2018), não incluindo os fármacos.

Na União Europeia, a aprovação de novos medicamentos requer a avaliação dos potenciais riscos ambientais associados à sua utilização, além da comprovação dos aspectos inerentes ao fármaco como segurança, estabilidade e eficácia. A Diretiva 2013/39/UE implementa programas de monitoramento para os contaminantes emergentes com a finalidade de priorizar as substâncias que apresentem risco para o ambiente aquático. O diclofenaco foi inserido na lista de vigilância de substâncias para as quais devem ser recolhidos, em toda a União Europeia, dados de monitoramento, uma vez que podem representar risco significativo para o meio aquático (PARLAMENTO EUROPEU, 2013).

\section{CONCLUSÃO}

A problemática ambiental da contaminação dos recursos hídricos pelos fármacos envolve grandes desafios, entre eles a busca por medidas que visem à prevenção de possíveis impactos no meio ambiente e na saúde humana. Os estudos sobre os efeitos aos seres não alvos são desenvolvidos com mais frequência, assim como os testes de ecotoxicidade, observando-se que os fármacos podem impactar na biota aquática por meio da feminização de peixes e diminuição da diversidade de espécies. Os estudos sobre os efeitos na saúde humana são recentes e escassos, sendo a maioria registrada na literatura internacional.
Outro desafio preocupante sobre essa problemática é a não regulamentação, ou seja, não há uma legislação que determine limites de lançamento para esse tipo de substância no meio ambiente, muito embora as discussões sobre o tema e as pesquisas têm contribuído significativamente, fornecendo inúmeros subsídios para tomadas de decisões. No entanto, a falta de regulamentações deixa lacunas para que esses contaminantes sejam lançados nos corpos d'água. Assim, o ideal é que estudos continuem sendo realizados e que medidas de prevenção sejam estudadas a fim de reduzir a presença desses contaminantes nas diferentes matrizes ambientais e, consequentemente, preservar a saúde da população e do meio ambiente.

\section{REFERÊNCIAS}

ALMEIDA, G. A.; WEBER, R. R. Fármacos na represa Billings. Ambiente e Saúde, Joinville, v. 6, n. 2, p. 7-13, 2006.

AMÉRICO, J. H. P.; ISIQUE, W. D.; MINILLO, A.; CARVALHO, S. L.; TORRES, N. H. Fármacos em uma estação de tratamento de esgoto na região Centro-oeste do Brasil e os riscos aos recursos hídricos. Revista Brasileira de Recursos Hídricos, Porto Alegre, v. 17, n. 3, p. 61-67, jul./set. 2012. http://dx.doi.org/10.21168/rbrh.v17n3.p61-67 
AMÉRICO-PINHEIRO, J. H. P.; ISIQUE, W. D.; TORRES, N. H.; MACHADO, A. A.; CARVALHO, S. L.; VALÉRIO FILHO, W. V.; FERREIRA, L. F. R. Ocorrência de diclofenaco e naproxeno em água superficial no município de Três Lagoas (MS) e a influência da temperatura da água na deteç̧ão desses anti-inflamatórios. Engenharia Sanitária e Ambiental, Rio de Janeiro, v. 22, n. 3, p. 429-235, maio/jun. 2017. http://dx.doi.org/10.1590/s1413-41522017128719

BILA, D. M.; DEZOTTI, M. Fármacos no meio ambiente. Química Nova, São Paulo, v. 26, n. 4, p. 523-530, 2003.

BISOGNIN, R. P.; WOLFF, D. B.; CARISSIMI, E. Revisão sobre fármacos no ambiente. Revista DAE, São Paulo. v. 66, n. 210, p. 78-95, 2018. http://dx.doi.org/10.4322/dae.2018.009

BRASIL. Conselho Nacional do Meio Ambiente (CONAMA). Resolução no 357, de 17 de marco de 2005. Dispõe sobre a classificação dos corpos de água e diretrizes ambientais para o seu enquadramento, bem como estabelece as condições e padrões de lançamento de efluentes, e dá outras providências. Diário Oficial da União, Brasília, 2005. Disponível em: <http://www.mma.gov.br/port/conama/res/res05/res35705.pdf>. Acesso em: 15 ago. 2018.

BRASIL. Conselho Nacional do Meio Ambiente (CONAMA). Resolução no 396, de 3 de abril de 2008. Dispõe sobre a classificação e diretrizes ambientais para o enquadramento das águas subterrâneas e dá outras providências. Diário Oficial da União, Brasília, 2008. Disponível em: <http://www.mma.gov.br/port/conama/legiabre. cfm?codlegi=562>. Acesso em: 10 jun. 2018.

BRASIL. Conselho Nacional do Meio Ambiente (CONAMA). Resolução no 430, de 13 de maio de 2011. Dispõe sobre as condições e padrões de lançamento de efluentes, complementa e altera a Resolução no 357, de 17 de março de 2005, do Conselho Nacional do Meio Ambiente - CONAMA. Diário Oficial da União, Brasília, 2011. Disponível em: <http://www.mma.gov.br/port/conama/legiabre.cfm?codlegi=646>. Acesso em: 10 jun. 2018.

CAMPANHA, M. B.; AWAN, A. T.; DE SOUSA, D. N.; GROSSELI, G. M.; MOZETO, A. A.; FADINI, P. S. A 3-year study on occurrence of emerging contaminants in an urban stream of São Paulo State of Southeast Brazil. Environmental Science and Pollution Research International, v. 22, n. 10, p. 7936-7947, 2015. https://doi.org/10.1007/s11356-014-3929-x

CUNHA, D. L.; PAULA, L. M.; SILVA, S. M. C.; BILA, D. M.; FONSECA, E. M.; OLIVEIRA, J. L. M. Ocorrência e remoção de estrogênios por processos de tratamento biológico de esgotos. Ambiente e Água, Taubaté, v. 12, n. 2, p. 249-262, 2017. http://dx.doi.org/10.4136/ambi-agua.1992

FENT, K.; WESTON, A. A.; CAMINADA, D. Ecotoxicology of human pharmaceuticals. Aquatic Toxicology, v. 76, n. 2, p. 122-159, 2006. https://doi.org/10.1016/j.aquatox.2005.09.009

GARRISON, A. W.; POPE, J. D.; ALLEN, F. R.; KEITH, C. H . Identification and analysis of organic pollutants in water. In: KEITH, C. H. (org.). Identification and Analysis of Organic Pollutants in Water. Michigan: Ann Arbor Science, 1976. p. 517-566.

GHELFI, A. Efeitos toxicológicos do diclofenaco em peixes Rhamdia quelen. 72f. Dissertação (Mestrado em Ecologia e Conservação) - Faculdade de Ciências Biológicas, Universidade Federal do Paraná, Curitiba, 2014. Disponível em: <https://acervodigital.ufpr.br/handle/1884/35405>. Acesso em: 20 jul. 2018.

GHISELLI, G.; JARDIM, W. Interferentes endócrinos no ambiente. Química Nova, São Paulo, v. 30, n. 3, p. 695-706, 2007.

GUILOSKI, I. C.; RIBAS, J. L. C.; PIANCINI, L. D. S.; DAGOSTIM, A. C.; CIRIO, S. M.; FÁVARO, L.F.; BOSCHEN, S. L.; CESTARI, M. M.; DA CUNHA, C.; SILVA DE ASSIS, H. C. Paracetamol causes endocrine disruption and hepatotoxicity in male fish Rhamdia quelen after subchronic exposure. Environmental Toxicology and Pharmacology, v. 53, p. 111-120, 2017. https://doi.org/10.1016/j.etap.2017.05.005

HOEGER, B.; KÖLLNER, B.; DIETRICH, D. R.; HITZFELD, B. Water-borne diclofenac affects kidney and gill integrity and selected immune parameters in brown trout (Salmo trutta f. fario). Aquatic Toxicology, v. 75, n. 1, p. 53-64, 2005. https://doi.org/10.1016/j.aquatox.2005.07.006 
KHETAN, S. K.; COLLINS, T. J. Human pharmaceuticals in the aquatic environment: a challenge to green chemistry. Chemical Reviews, v. 107, n. 6, p. 2319-2364, 2007. https://doi.org/10.1021/cr020441w

KRAMER, R. D.; MIZUKAWA, A.; IDE, A. H.; MARCANTE, L. O.; SANTOS, M. M.; AZEVEDO, J. C. R. Determinação de anti-inflamatórios na água e sedimento e suas relações com a qualidade da água na bacia do Alto Iguaçu, Curitiba-PR. Revista Brasileira de Recursos Hídricos, Porto Alegre, v. 20, n. 3, p. 657-667, jul./set. 2015. http://dx.doi.org/10.21168/ rbrh.v20n3.p657-667

KÜMMERER, K. Pharmaceuticals in the environment. The Annual Review of Environment and Resources, v. 35, p. 57-75, 2010. https://doi.org/10.1146/annurev-environ-052809-161223

NAVA-ÁlvareZ, R.; RAZO-ESTRAdA, A. C.; MEDINA, S. G.; GÓMEZ-OlivÁN, L. M.; MARTíNEZ, M. G. Oxidative Stress Induced by Mixture of Diclofenac and Acetaminophen on Common Carp (Cyprinus carpio). Water, Air, and Soil Pollution, v. 225, n. 2, p. 1-9, 2014. http://dx.doi.org/10.1007/s11270-014-1873-5

PARLAMENTO EUROPEU. Diretiva 2013/39/UE do Parlamento Europeu e do Conselho de 12 de agosto de 2013. Altera as Diretivas 2000/60/CE e 2008/105/CE no que respeita às substâncias prioritárias no domínio da política da água. Jornal Oficial da União Europeia, 2013. Disponível em: <https://eur-lex.europa.eu/legal-content/PT/TXT/ PDF/?uri=CELEX:32013L0039\&from=IT>. Acesso em: 16 ago. 2018.

PAROLINI, M.; BINELLI, A.; PROVINI, A. Chronic effects induced by ibuprofen on the freshwater bivalve Dreissena polymorpha. Ecotoxicology Environmental Safety, v. 74, n. 6, p. 1586-1594, 2011. https://doi.org/10.1016/j. ecoenv.2011.04.025

QUINN, B.; SCHMIDT, W.; O'ROURKE, K.; HERNAN, R. Effects of the pharmaceuticals gemfibrozil and diclofenac on biomarker expression in the zebra mussel (Dreissena polymorpha) and their comparison with standardized toxicity tests. Chemosphere, v. 84, n. 5, p. 657-663, 2011. https://doi.org/10.1016/j.chemosphere.2011.03.033

RAGUGNETTI, M.; ADAM, M. L.; GUIMARÃES, A. T. B.; SPONCHIADO, G.; VASCONCELOS, E. C.; OLIVEIRA, C. M. R. Ibuprofen genotoxicity in aquatic environment: an experimental model using Oreochromis niloticus. Water, Air, and Soil Pollution, Berlim, v. 218, n. 1, p. 361-364, 2011. https://doi.org/10.1007/s11270-010-0698-0

SANTOS, J. S. T. Resíduos de medicamentos: Riscos associados e comportamento da população portuguesa. 84f. Dissertação (Mestrado Integrado em Ciências Farmacêuticas) - Universidade Fernando Pessoa, Porto, 2014. Disponível em: <https://bdigital.ufp.pt/bitstream/10284/4311/1/PPGJoana\%20Santos.pdf>. Acesso em: 20 jul. 2018.

SANTOS, L. H.; ARAÚJO, A. N.; FACHINI, A.; PENA, A.; DELERUE-MATOS, C.; MONTENEGRO, M. C. Ecotoxicological aspects related to the presence of pharmaceuticals in the aquatic environment. Journal of Hazardous Materials, v. 175, n. 1-3, p. 45-95, 2010. https://doi.org/10.1016/j.jhazmat.2009.10.100

STELATO, E. S.; OLIVEIRA, T. G.; STUNGES, G. M.; SILVA, E. C. P.; CUBA, R. M. F.; MINILLO, A.; ISIQUE, W. D. Avaliação da presença de resíduos de anti-inflamatórios não esteroides nos córregos veado e cedro do município de Presidente Prudente (SP), Brasil. Revista Brasileira de Ciências Ambientais, n. 39, p. 97-113, 2016. https://doi.org/10.5327/Z2176-947820160050

STUMPF, M.; TERNES, T. A.; WILKEN, R. D.; RODRIGUES, S. V.; BAUMANN, W. Polar drug residues in sewage and natural waters in the state of Rio de Janeiro, Brazil. Science of the Total Environment, v. 225, n. 1-2, p. 135-141, 1999.

TORRES, N. H.; AMÉRICO, J. H. P.; FERREIRA, L. F. R.; NAZATO, C.; MARANHO, L. A.; VILCA, F. Z.; TORNISIELO, V. L. Fármacos no ambiente - Revisão. Revista de Estudos Ambientais, v. 14, n. 4, p. 67-75, jul./dez. 2012.

TRIEBSKORN, R.; CASPER, H.; SCHEIL, V.; SCHWAIGER, J. Ultrastructural effects of pharmaceuticals (carbamazepine, clofibric acid, metoprolol, diclofenac) in rainbow trout (Oncorhynchus mykiss) and common carp (Cyprinus carpio). Analytical and Bioanalytical Chemistry, v. 387, n. 4, p. 1405-1416, 2007. https://doi.org/10.1007/s00216-006-1033-x 
UNITED STATE ENVIRONMENTAL PROTECTION AGENCY (USEPA). Drinking Water Contaminant Candidate List (CCL) and Regulatory Determination. Estados Unidos: USEPA. Disponível em: <https://www.epa.gov/ccl>. Acesso em: 16 ago. 2018.

WILKINSON, J. L.; HOODA, P. S.; BARKER, J.; BARTON, S.; SWINDEN, J. Ecotoxic pharmaceuticals, personal care products, and other emerging contaminants: A review of environmental, receptor-mediated, developmental, and epigenetic toxicity with discussion of proposed toxicity to humans. Critical Reviews in Environmental Science and Technology, v. 46, n. 4, p. 336-381, 2015. https://doi.org/10.1080/10643389.2015.1096876

ZHANG, Y.; GEISSEN, S.; GAL, C. Carbamazepine and diclofenac: Removal in wastewater treatment plants and occurrence in water bodies. Chemosphere, v. 73, p. 1151-1161, 2008 https://doi.org/10.1016/j.chemosphere.2008.07.086 